\title{
Testing QCD with CMS using jets and diffraction
}

\author{
Hans Van Haevermaet* \\ University of Antwerp (BE) \\ E-mail: hans.van.haevermaetecern.ch
}

\begin{abstract}
Measurements of jet final states are presented, performed with the CMS experiment at the LHC at different centre-of-mass energies and down to very low $p_{\mathrm{T}}$. The study of forward and/or low$p_{\mathrm{T}}$ jets is ideal to explore new kinematic domains in QCD, especially in the low- $x$ region. A measurement of the production of leading charged particles, and leading charged-particle jets, at small $p_{\mathrm{T}}$ in proton-proton collisions at $\sqrt{s}=8 \mathrm{TeV}$ is presented, together with a first observation at the LHC of events with a large rapidity gap between the two leading jets at $\sqrt{s}=7 \mathrm{TeV}$.
\end{abstract}

The European Physical Society Conference on High Energy Physics 22-29 July 2015

Vienna, Austria

* Speaker. 


\section{Introduction}

The description of proton-proton ( $p p$ ) collisions at the Large Hadron Collider (LHC) is a nontrivial task that consists of several key elements. At its core is the usage of collinear factorisation [1], which states that a $p p$ interaction can be factorised in a hard scattering parton-parton cross section convolved with process independent parton density functions (PDFs). Where the former is a process dependent, short distance interaction with small coupling $\alpha_{S}$, that can be calculated using fixed order perturbation theory; the latter covers non-perturbative long distance interactions with a high coupling. The PDFs thus need to be measured in data at specific values of $x$ and $Q^{2}$, and the evolution in $x$ and $Q^{2}$ is then driven by the DGLAP equations.

This approach has been successful in describing many inclusive processes, however, it is only valid for one hard momentum scale and not too low values of $x$. Furthermore, the treatment of initial transverse momenta of the partons is neglected in this framework. One also knows from measurements performed at hadron colliders, that the above picture needs to be complemented with phenomenological models to describe the data. In particular, the addition of initial and final state radiation, and multiple parton interactions (MPI) is crucial.

With the LHC operating at centre-of-mass energies of $7 \mathrm{TeV}, 8 \mathrm{TeV}$, and recently $13 \mathrm{TeV}$, one has the unique ability to probe new kinematic regions in $x$ and $Q^{2}$. It allows us to find out if the collinear approximations stay valid at the LHC, or that there is a need to include $k_{\mathrm{T}}$ dependent, unintegrated PDFs. To check wether the evolution of the PDFs can still be described by DGLAP, or that alternative approximations, like e.g. BFKL or CCFM, take over. Furthermore, gluons dominate the low- $x$ region [2], which could imply the appearance of saturation or recombination effects at the LHC. In addition it is also important to fully understand the role of MPI in the description of $p p$ collisions.

To probe the new kinematic regions available at the LHC, in particular the low- $x$ region, one can use forward and/or low- $p_{\mathrm{T}}$ jets: $x \sim\left(p_{\mathrm{T}} / \sqrt{s}\right) e^{-|\eta|}$. The CMS experiment [3] is ideal to study such final state objects thanks to its extensive calorimetric coverage, up until $-6.6<\eta<5.2$, and its precise central tracking detector that is able to measure very low $p_{\mathrm{T}}$ charged particles. During the LHC Run I, CMS performed several measurements (e.g. [4-6]) to study forward and/or low$p_{\mathrm{T}}$ jets, involving various final state configurations. In these proceedings we will address in more detail two recent results: the study of leading charged particles and jets at small $p_{\mathrm{T}}$ [7]; and the study of di-jet production with a large rapidity gap [8].

\section{Leading charged particles and jets at small $p_{\mathbf{T}}$}

A study of the production of leading charged particles, and leading charged-particle jets, at small $p_{\mathrm{T}}$ in $p p$ collisions at $\sqrt{s}=8 \mathrm{TeV}$ is presented [7]. It is sensitive to the momentum scale at which parton densities saturate, and to the behaviour of MPI. In addition it allows one to probe the transition from the perturbative to non-perturbative region [9]. In $p p$ collisions most particles originate from non-pQCD semi-hard interactions $\left(p_{\mathrm{T}} \sim 1-3 \mathrm{GeV}\right)$, and the partonic cross section diverges at low $p_{\mathrm{T}}: d \sigma / d p_{\mathrm{T}}^{2} \propto \alpha_{S}^{2}\left(p_{\mathrm{T}}\right) / p_{\mathrm{T}}^{4}$ [10]. It will therefore exceed the total inelastic $p p$ cross section and needs to be tamed, a feature that can be explored in this analysis. 
The aim is to look at the per-event yield of leading charged particles, and charged-particle jets integrated above a given $p_{\mathrm{T}}^{\min }$, with $p_{\mathrm{T}}^{\min }>0.8$ and $1.0 \mathrm{GeV}$ respectively:

$$
r\left(p_{\mathrm{T}}^{\min }\right)=\frac{1}{N_{\mathrm{evt}}} \int_{p_{\mathrm{T}}^{\min }} d p_{\mathrm{T}}^{\text {lead }}\left(\frac{d N}{d p_{\mathrm{T}}^{\text {lead }}}\right)
$$

with $N_{\text {evt }}$ the number of events with a leading charged particle with $p_{\mathrm{T}}>0.4 \mathrm{GeV}$ and $|\eta|<2.4$; and $N$ the number of events with a leading charged particle $(|\eta|<2.4)$ or leading jet $(|\eta|<1.9$, anti- $k_{\mathrm{T}} \mathrm{R}=0.5$ ) with transverse momentum $p_{\mathrm{T}}^{\text {lead }}$.

The results are shown in fig. 1 for leading charged particles. Since our goal is to study the very low $p_{\mathrm{T}}$ region, the Monte Carlo predictions are scaled to data at $p_{\mathrm{T}}^{\min }=9 \mathrm{GeV}$. In the left figure, data are compared with various configurations of the PYTHIA6 Monte Carlo event generator. This model uses a phenomenological approach, by introducing a $p_{\mathrm{T}, 0}$ cut-off parameter $1 / p_{\mathrm{T}}^{4} \rightarrow 1 /\left(p_{\mathrm{T}}^{2}+p_{\mathrm{T}, 0}^{2}\right)^{2}$, to regularise the partonic cross section [11]. When this mechanism is turned off, a clear discrepancy of more than one order of magnitude appears, which indicates the necessity of the taming of the cross section. The effect of MPI on the other hand, is small. In addition, the distributions show that the transition from the perturbative to the non-perturbative regime happens in the $O(1-5 \mathrm{GeV})$ region. From all compared models, EPOS describes the data best.
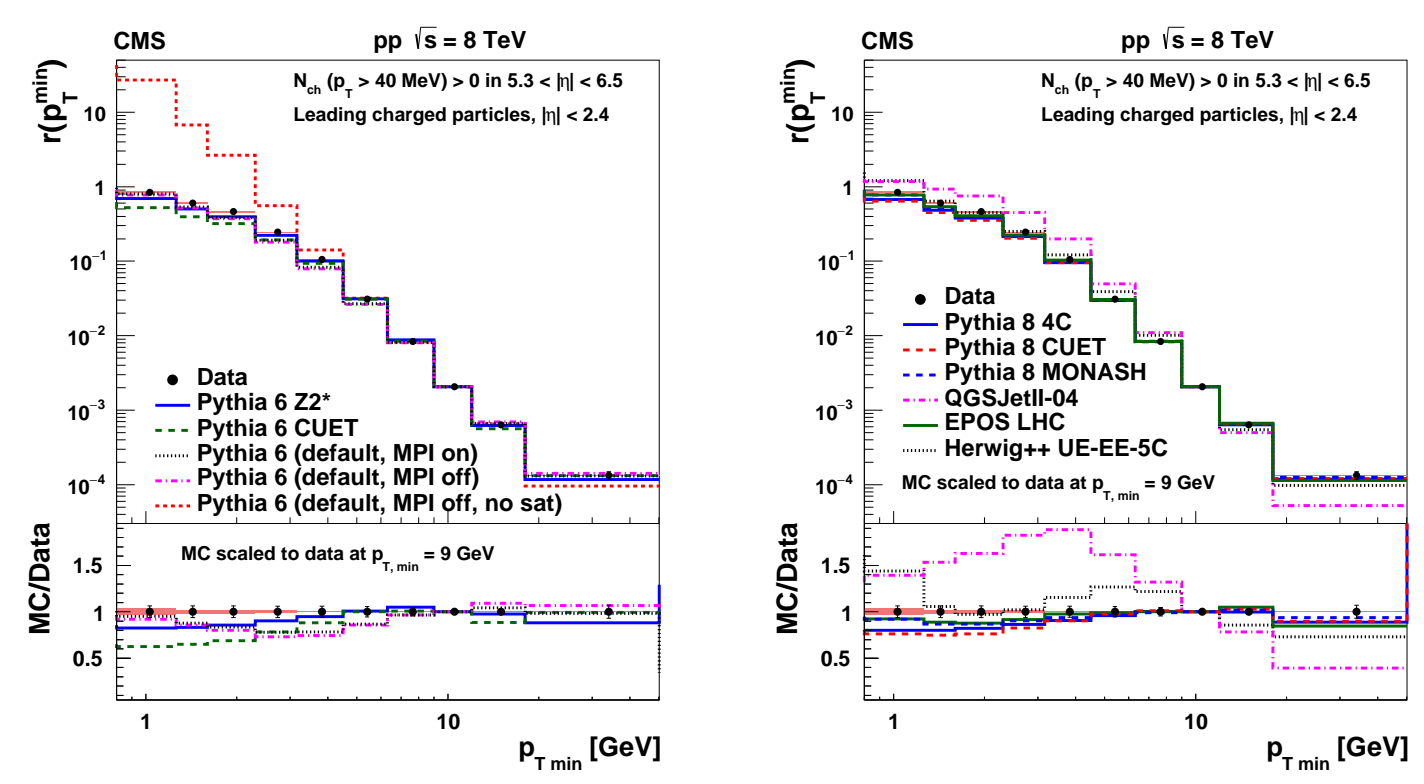

Figure 1: The integrated yield, $r\left(p_{\mathrm{T}}^{\min }\right)$, of events with a leading charged particle within $|\eta|<2.4$ as a function of $p_{\mathrm{T}}^{\min }$. Data are compared to predictions from several PYTHIA6 tunes (left), and various other Monte Carlo event generators (right). The predictions are scaled to the measured value of $r\left(p_{\mathrm{T}}^{\text {lead }}>9.0 \mathrm{GeV}\right)$. The prediction from PYTHIA6 with MPI off and no parton saturation is not shown in the MC/data ratio panel because of the large disagreement with the data. [7]

Fig. 2 shows the results for leading charged-particle jets, for which the Monte Carlo predictions are scaled to data at $p_{\mathrm{T}}^{\min }=14.3 \mathrm{GeV}$. In the left figure data are again compared to different PYTHIA6 configurations. Same conclusions are valid, except that the description at low $p_{\mathrm{T}}$ is worse, 
which is due to the influence of MPI in the jet cone. Taking all models in fig. 2 into account, here too EPOS describes the data best.
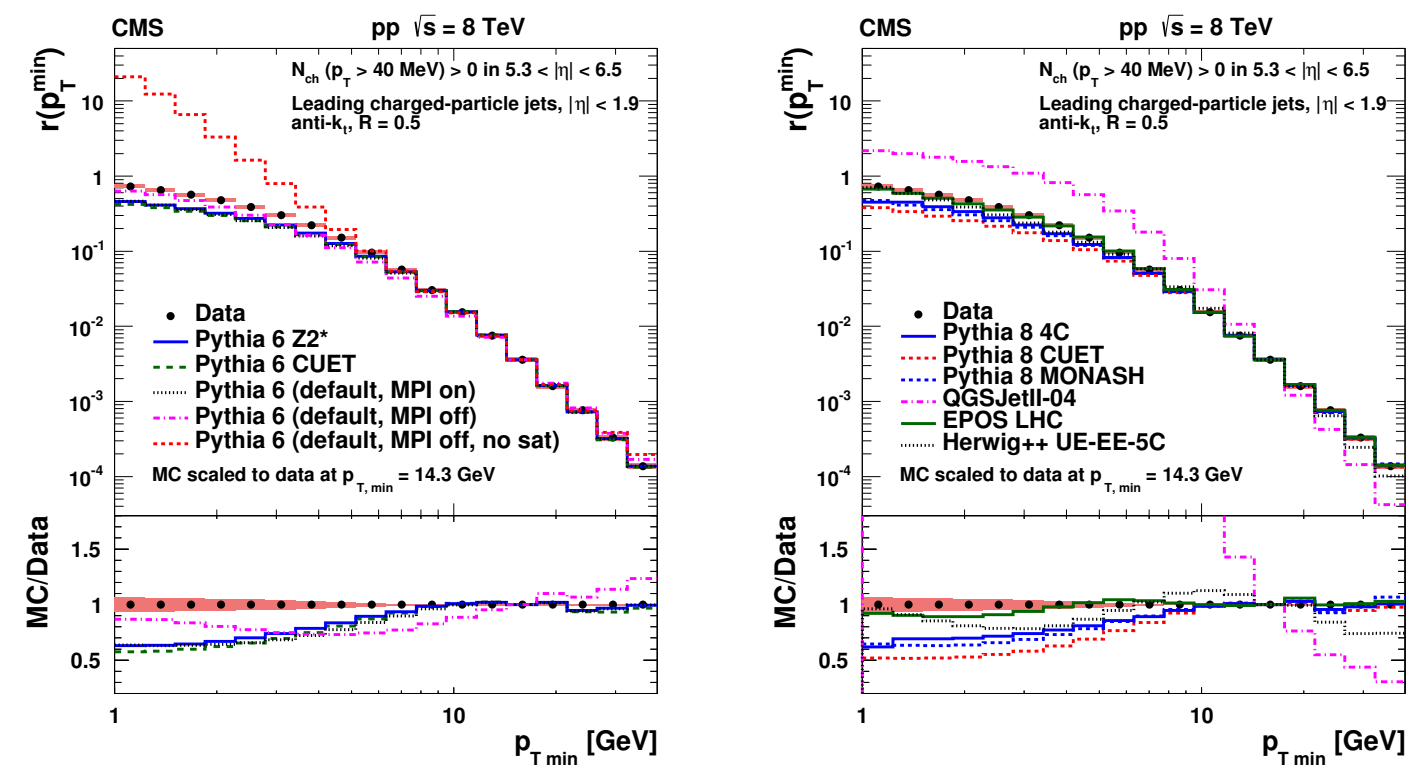

Figure 2: The integrated yield, $r\left(p_{\mathrm{T}}^{\min }\right)$, of events with a leading charged-particle jet within $|\eta|<1.9$ as a function of $p_{\mathrm{T}}^{\mathrm{min}}$. Data are compared to predictions from several PYTHIA6 tunes (left), and various other Monte Carlo event generators (right). The predictions are scaled to the measured value of $r\left(p_{\mathrm{T}}^{\text {lead }}>14.3 \mathrm{GeV}\right)$. The prediction from PYTHIA6 with MPI off and no parton saturation is not shown in the MC/data ratio panel because of the large disagreement with the data. [7]

\section{Di-jet production with a large rapidity gap}

A measurement of di-jet production with a large rapidity gap between the jets is presented at a centre-of-mass energy of $\sqrt{s}=7 \mathrm{TeV}$ [8]. It is a diffractive process in which a colour singlet exchange (CSE) takes place between the incoming partons. However, the four-momentum squared carried in such events is significantly larger than in standard diffractive processes. These CSE events can be understood in a BFKL inspired perturbative QCD approach in terms of the exchange of a colour singlet gluon ladder. The study of CSE events thus may allow one to disentangle BFKL dynamics from DGLAP evolution, and is also sensitive to the contribution from rescattering processes that can destroy the rapidity gap.

A first observation of jet-gap-jet events at the LHC is presented, and the aim of the analysis is to extract the fraction of CSE events, $f_{\mathrm{CSE}}$, as a function of $p_{\mathrm{T}}^{\text {jet2 }}$, the transverse momentum of the second leading jet in the event, and $\Delta \eta_{\mathrm{jj}}$, the difference in pseudorapidity between the two leading jets in the event. The jets are required to have $p_{\mathrm{T}}>40 \mathrm{GeV}$ and $1.5<|\eta|<4.5$, reconstructed in opposite hemispheres of the CMS detector. The fraction $f_{\mathrm{CSE}}$ is extracted from the charged particle multiplicity distribution, for tracks with $p_{\mathrm{T}}>0.2 \mathrm{GeV}$, in a fixed region of $|\eta|<1$ between the jets. Jet-gap-jet events with a colour singlet exchange then show up in the charged particle multiplicity 
distribution as an excess of events with no or very low multiplicity. This is confirmed in fig. 3 , which shows the multiplicity distribution for the lowest $p_{\mathrm{T}}^{\text {jet2 }}$ bin. The data are described by a PYTHIA6 model (LO DGLAP, with no CSE contributions) with exception of the lowest multiplicity bins, where an excess of events is observed, indicating a contribution from CSE events, which is well described by the HERWIG6 model (LL BFKL).

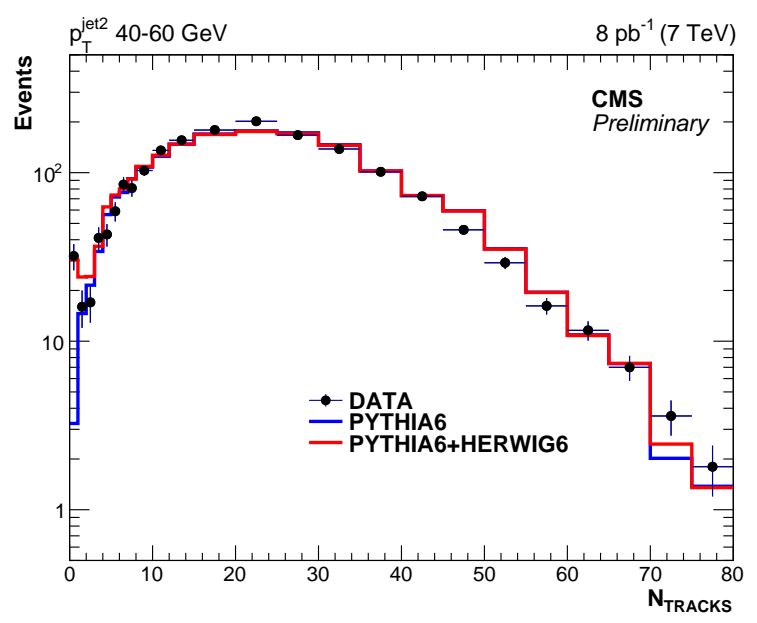

Figure 3: Charged particle multiplicity distribution for $40<p_{\mathrm{T}}^{\text {jet2 }}<60 \mathrm{GeV}$, compared to predictions of PYTHIA6 and HERWIG6 event generators. The normalisation of the MC distributions is obtained from a fit to the data. [8]

The fraction $f_{\mathrm{CSE}}$ is then defined as:

$$
f_{\mathrm{CSE}}=\frac{N_{\text {events }}(\mathrm{S})-N_{\mathrm{bkg}}(\mathrm{S})}{N_{\text {events }}},
$$

with $N_{\text {events }}(\mathrm{S})$ the number of events in the first $\mathrm{S}$ bins of the multiplicity distribution, $N_{\mathrm{bkg}}(\mathrm{S})$ the estimated number of events in these bins originating from non-CSE events, and $N_{\text {events }}$ the total number of events in the distribution. While $N_{\text {events }}(\mathrm{S})$ and $N_{\text {events }}$ are straightforward to extract, the determination of $N_{\mathrm{bkg}}(\mathrm{S})$ requires a more careful method. Two approaches are used [8], in the first the shape of the background is obtained from a sample where the two leading jets are required to be on the same side (SS) of the detector, and the second approach is based on a fit of the multiplicity distribution with a negative binomial distribution (NBD) function. The two methods produce consistent results of the background estimation. The NBD fit is chosen as default, and the SS method is used as systematic check.

The results as a function of $p_{\mathrm{T}}^{\text {jet2 }}$ are shown in fig. 4, and compared to data from other experiments (left) and the Mueller \& Tang model [12] (right). One sees a modest increase of $f_{\mathrm{CSE}}$ with $p_{\mathrm{T}}^{\text {jet2 }}$ and a suppression of the gap fraction with increasing centre-of-mass energy. For $40<p_{\mathrm{T}}^{\text {jet2 }}<$ $60 \mathrm{GeV}$ the value at $\sqrt{s}=7 \mathrm{TeV}$ is a factor $\sim 2$ lower than at $\sqrt{s}=1.8 \mathrm{TeV}$, which can be due to stronger contributions from rescattering processes. The Mueller \& Tang model does not reproduce the rise with $p_{\mathrm{T}}^{\mathrm{jet} 2}$ and underestimates $f_{\mathrm{CSE}}$.

Fig. 5 shows the results as function of $\Delta \eta_{\mathrm{jj}}$, the difference in pseudorapidity between the two leading jets, for the three $p_{\mathrm{T}}^{\text {jet} 2}$ bins. Here CMS data are compared to the Mueller \& Tang model, 

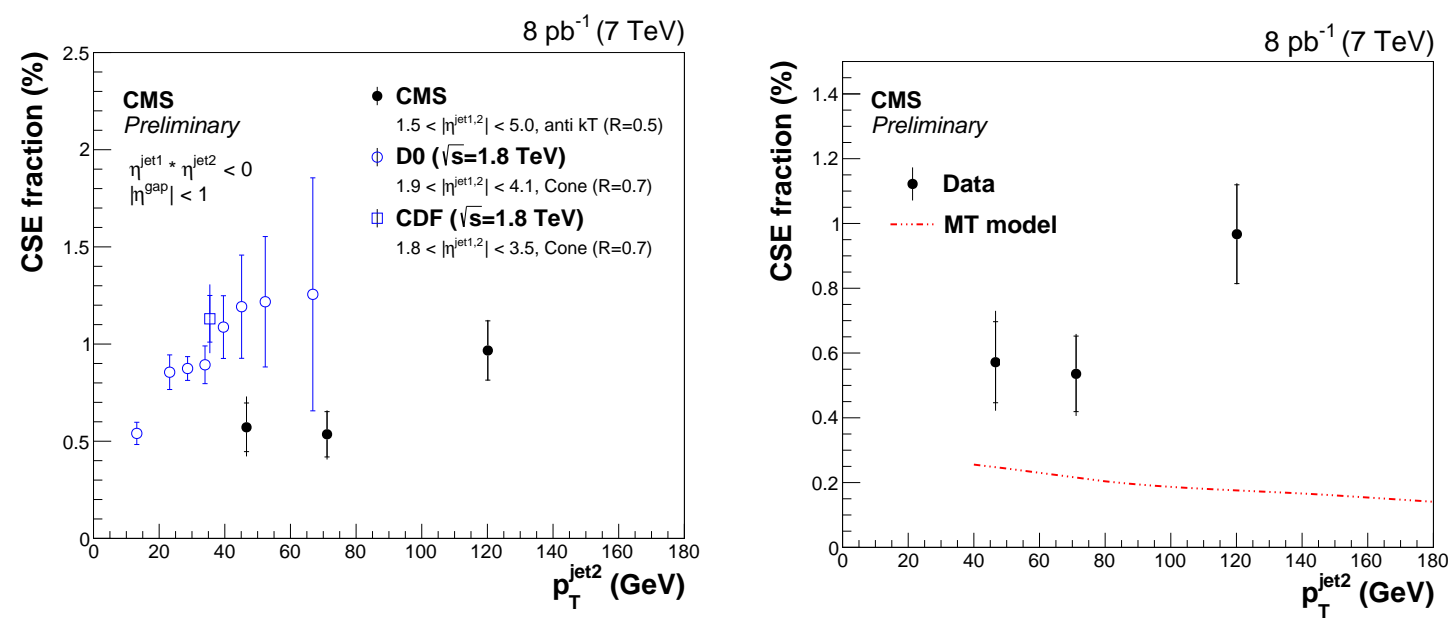

Figure 4: The fraction $f_{\mathrm{CSE}}$ as a function of $p_{\mathrm{T}}^{\text {jet2 }}$ as measured by CMS for $40<p_{\mathrm{T}}^{\text {jet2 }}<60 \mathrm{GeV}, 60<p_{\mathrm{T}}^{\text {jet2 }}<$ $100 \mathrm{GeV}$, and $100<p_{\mathrm{T}}^{\text {jet } 2}<200 \mathrm{GeV}$ at $\sqrt{s}=7 \mathrm{TeV}$, compared to results of D0 and CDF at $\sqrt{s}=1.8 \mathrm{TeV}$ (left), and to the Mueller \& Tang (MT) model (right). The results are plotted at the mean value of $p_{\mathrm{T}}^{\text {jet2 }}$ in the bin. [8]

which does not reproduce the rise with $\Delta \eta_{\mathrm{jj}}$ and underestimates the fraction $f_{\mathrm{CSE}}$, specifically at higher $p_{\mathrm{T}}^{\mathrm{jet} 2}$ values.

\section{Summary}

The CMS experiment has extensively studied new kinematic regions of QCD in proton-proton collisions, available at the LHC with data from Run I at various centre-of-mass energies. In particular, several measurements use forward and/or low- $p_{\mathrm{T}}$ jet final states to probe the low- $x$ region. The production of leading charged particles, and leading charged-particle jets, at small $p_{\mathrm{T}}$ in $p p$ collisions at $\sqrt{s}=8 \mathrm{TeV}$ is presented, and shows that the influence of the phenomenological dampening factor is clear. In addition, a first observation of jet-gap-jet events with a colour singlet exchange at the LHC is shown. The fraction of CSE events is successfully extracted and compared to data from other experiments, and the Mueller \& Tang model. The fraction of CSE events is, as expected, suppressed at $\sqrt{s}=7 \mathrm{TeV}$ with respect to lower centre-of-mass energies, and consistent with the Tevatron data.

\section{References}

[1] J. C. Collins, D. E. Soper and G. F. Sterman, Adv. Ser. Direct. High Energy Phys. 5 (1989) 1 [hep-ph/0409313].

[2] F. D. Aaron et al. [H1 and ZEUS Collaborations], JHEP 1001 (2010) 109 [arXiv:0911.0884 [hep-ex]].

[3] S. Chatrchyan et al. [CMS Collaboration], JINST 3 (2008) S08004.

[4] CMS Collaboration [CMS Collaboration], CMS-PAS-FSQ-12-031.

[5] CMS Collaboration [CMS Collaboration], CMS-PAS-FSQ-12-008.

[6] CMS Collaboration [CMS Collaboration], CMS-PAS-FSQ-12-002. 

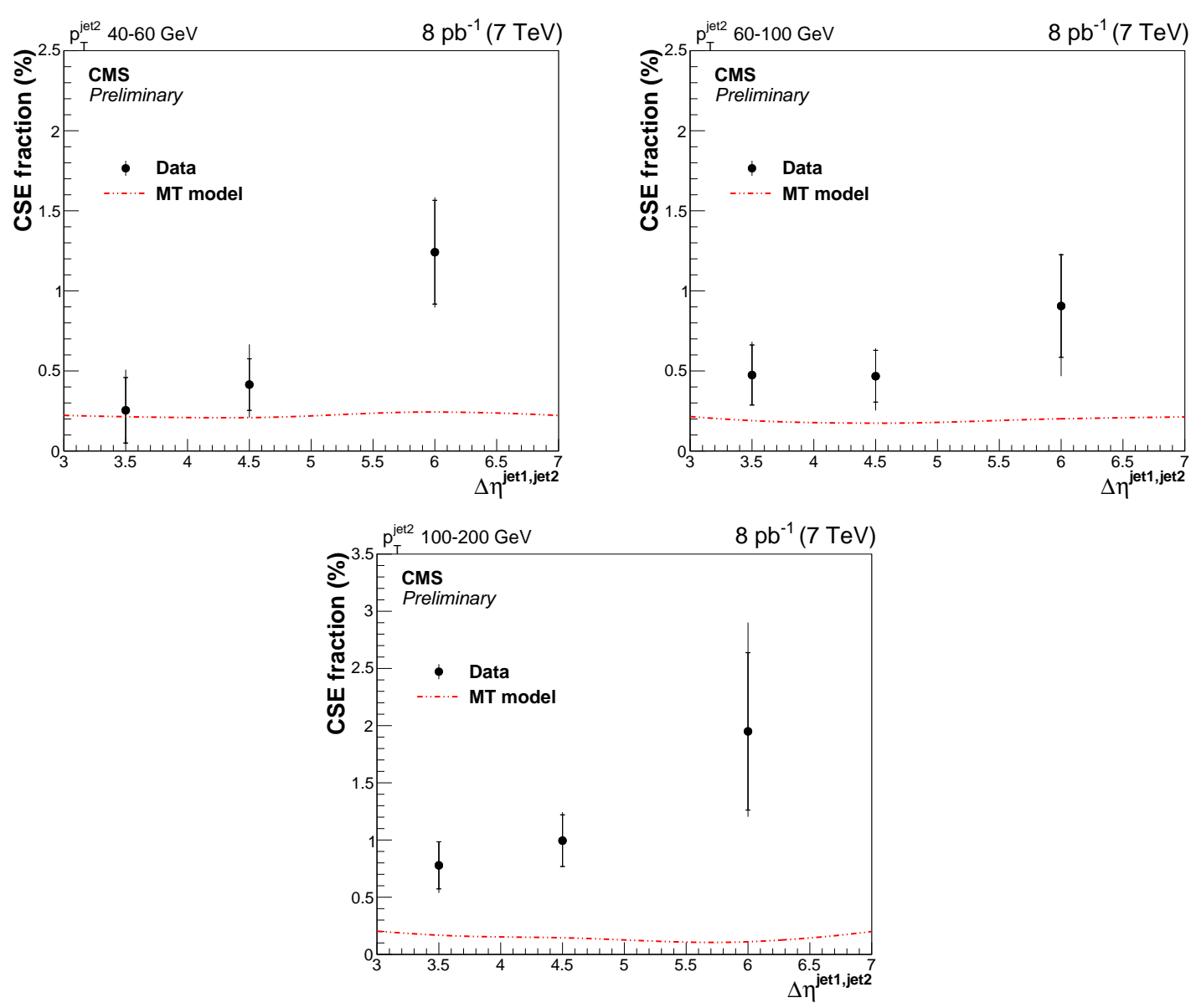

Figure 5: The fraction $f_{\mathrm{CSE}}$ as a function of $\Delta \eta_{\mathrm{jj}}$ measured in three bins of $p_{\mathrm{T}}^{\text {jet2 }}: 40-60 \mathrm{GeV}, 60-100 \mathrm{GeV}$, and 100-200 GeV, compared to predictions from the Mueller \& Tang (MT) model. The results are plotted at the center of each $\Delta \eta_{\mathrm{jj}}$ bin. [8]

[7] V. Khachatryan et al. [CMS Collaboration], arXiv:1507.00233 [hep-ex].

[8] CMS Collaboration [CMS Collaboration], CMS-PAS-FSQ-12-001.

[9] A. Grebenyuk, F. Hautmann, H. Jung, P. Katsas and A. Knutsson, Phys. Rev. D 86 (2012) 117501 [arXiv:1209.6265 [hep-ph]].

[10] T. Sjostrand and M. van Zijl, Phys. Rev. D 36 (1987) 2019.

[11] T. Sjostrand and P. Z. Skands, JHEP 0403 (2004) 053 [hep-ph/0402078].

[12] A. H. Mueller and W. K. Tang, Phys. Lett. B 284 (1992) 123. 